\title{
ATOMIC FORCE MICROSCOPE FOR PLANETARY APPLICATIONS
}

\author{
T. Akiyama, S. Gautsch, N.F. de Rooij, U. Staufer \\ Institute of Microtechnology, Univ. of Neuchâtel, Jaquet-Droz 1, 2007 Neuchâtel, Switzerland. \\ Ph. Niedermann \\ CSEM, Jaquet-Droz 1, 2007 Neuchâtel, Switzerland. \\ L. Howald, and D. Müller \\ Nanosurf AG, Austrasse 4, 4410 Liestal, Switzerland. \\ A. Tonin, and H.-R. Hidber \\ Insitute of Physics, Univ. of Basel, Klingelbergstr . 824056 Basel, Switzerland \\ W. T. Pike, M. H. Hecht \\ Jet Propulsion Laboratory, 4800 Oak Grove Dr. Pasadena, CA 91109-8099, USA
}

\begin{abstract}
We have developed, built and tested an atomic force microscope (AFM) for planetary science applications, in particular for the study of Martian dust and soil. The system consists of a controller board, an electromagnetic scanner and a microfabricated sensor-chip. Eight cantilevers with integrated, piezoresistive deflection sensors are aligned in a row and are engaged one after the other to provide redundancy in case of tip or cantilever failure. Silicon and molded diamond tips are used for probing the sample. Images can be recorded in both, static and dynamic operation mode. In the latter case, excitation of the resonance frequencies of the cantilevers is achieved by vibrating the whole chip with a piezoelectric disk.
\end{abstract}

\section{INTRODUCTION}

Recent data $[1,2]$ from both Mars Pathfinder and Mars Global Surveyor indicate a vigorous recirculation of dust between the Martian surface and atmosphere. Dust devils scour the ground, leaving myriad dark trails, lofted material colors the sky, and global dust storms envelop the planet. Althongh properties of the dust have been inferred from remote sensing, there has been no imaging of individual dust and soil particles to determine their size distribution and shape. Such information is essential in both understanding the contribution of the particles to the Martian dynamics, and assessing the harmful effects of the dust on both robotic and human missions to the surface of Mars. NASA's Mars Environmental Compatibility Assessment (MECA) payload, which

a)

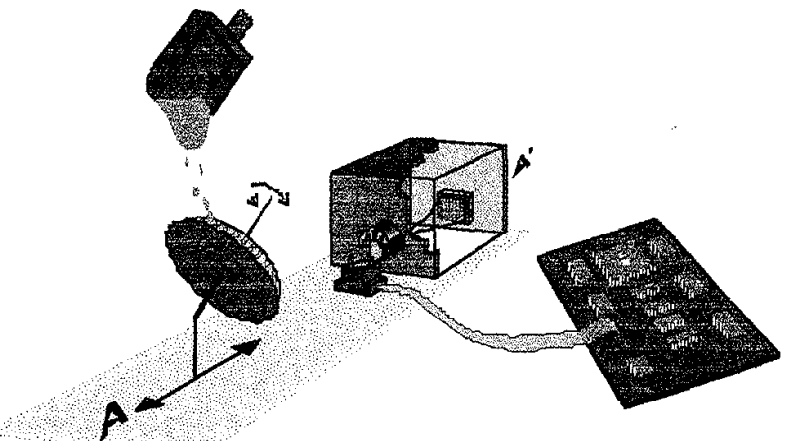

Figure 1 Set-up of the microscopy experiment of NASA's 2001 lander( $a$ ). A rotating sample stage delivers the soil collected by the robotic arm and brings it in front of the optical microscope and the AFM. 16 shows a photograph of the actual sample stage, AFM scanner and optical microscope. will be sent to Mars in the next three years, contains a microscopy station to produce images of dust and soil particles. Mars Pathfinder data indicates that the mean particle size of Martian atmospheric dust is less than 2 micrometers. Hence MECA's microscopy station, in addition to an optical microsope capable of taking color and ultraviolet fluorescent images, includes an AFM to image far below optical resolution (fig. 1). The sample-handling system consists of an external robot arm, for delivery of surface and subsurface soil samples, and a two-degree-of-freedom stage. The stage contains 69 substrates that can be rotated in turn to the field of view of the two microscopes, and then translated for focussing and AFM approach in $0.25 \mu \mathrm{m}$ steps.

The AFM is well-established instruments for imaging conductive and insulating samples below the resolution limit of optical microscopes in laboratories and production environment. A sharp tip mounted on a thin cantilever beam is brought into close proximity to the sample surface. Forces acting between the sample and the probe deflect the cantilever. This deflection is monitored and provides the topographic image of the sample. Alternatively, the cantilever is excited at its resonance frequency. This resonance is de-tuned when exposed to the force gradient above the sample surface. This signal can be used for imaging the sample in a comparable way as the lever bending in the above-mentioned static mode. This second, dynamic mode has the advantage that lateral forces between the tip and the sample are minimized. In the imaging of particles dynamic mode is therefore preferred as it has been found that the tip does not push the particles around during scanning.

The requirements on an AFM for space applications and

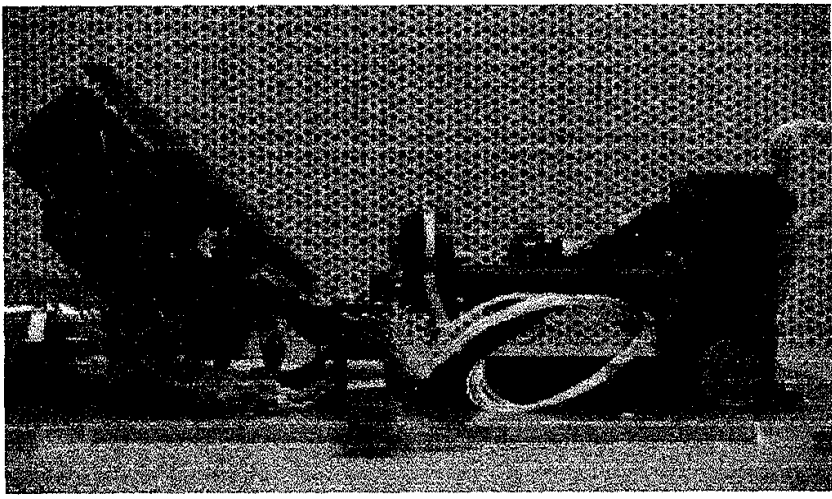

\section{b)}




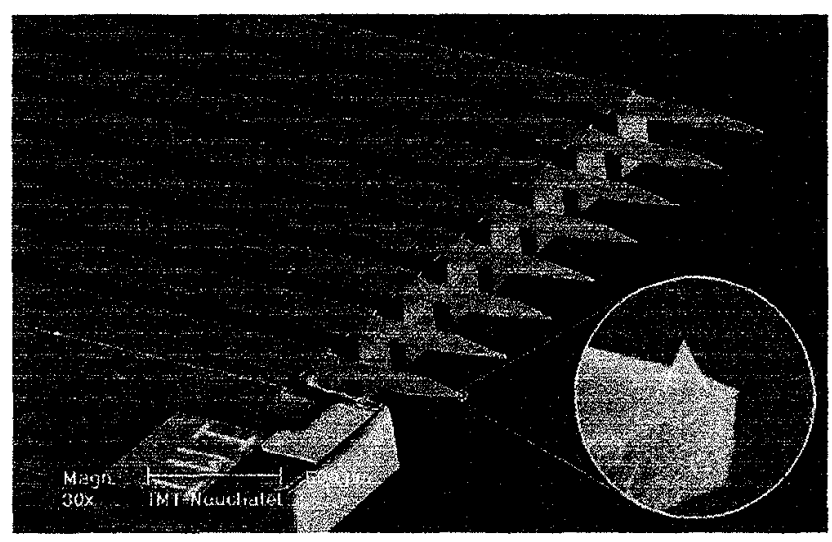

Figure 2. SEM picture of the microfabricated AFM chip with support beams etched by DRIE. The inset shows the silicon tip of cantilever 8. The CVD molded diamond tip can be viewed in fig 5. On the bottom part of the picture, the thermal compensation or with its protection bar is shown.

planetary research are quite different compared to those for laboratory use. Most important are weight and volume limitations, operation temperatures and shock and vibration conditions during launch and landing of the space vehicle. The electronic controller must be radiation hard or, at least, radiation tolerant. Moreover, there will be no operator for optimizing measuring parameters or tip exchange. Hence the AFM should be capable of self initialization, operate well under a broad range of conditions, and be capable of autonomous tip exchange, all within tight payload constraints.

\section{AFM DESIGN AND FABRICATION}

Integration from the initial design of the AFM with the optical microscope and sample-handing hardware, together with the development of an intelligent control system were necessary in achieving the required performance within the system constraints. In addition, maximum functionality was transferred to a microfabricated implementation of the AFM (fig. 2). First, the stress sensor is integrated into the micro-fabricated silicon cantilever. Second, means for exchanging blunt tips or broken cantilevers is provided through the use of a cantilever array. Third, diamond was incorporated as a low-wear material for selected tips in the array. Through this concentration of functionality into the microfabricated component of the instrument, constraints could be relaxed for the other subsystems; the scanner and servomotor as well as the electronic controller could be build with conventional technology, albeit tailored to perform reliably in the expected environments of the mission.

The cantilever deflection is measured by means of implanted piezoresistors in a Wheatstone bridge configuration [3] A special reference resistor is incorporated on an ultra-short cantilever for compensating thermal drifts. This reference cantilever is protected against mechanical damage by a surrounding, rigid safety bar. Electrical isolation between the resistors and the cantilevers is achieved by reverse biasing the pn-junction formed between the ptype resistors and the n-type bulk of the cantilever. This limits, to some extent, the range of usable tip-potential which can only be

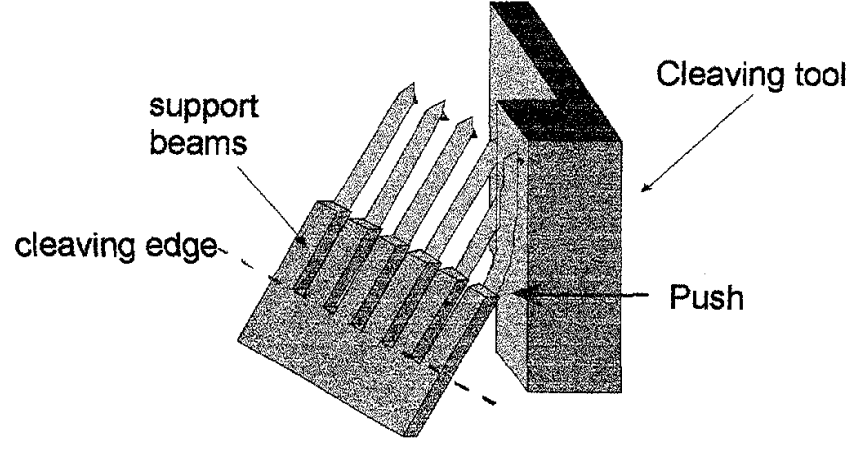

Figure 3. Method for removal of damaged cantilevers and support beams.

applied via the cantilever. It is expected that in the dry Martian atmosphere electrostatic forces between the sample and the tip will be present. The ability to change the tip potential is therefore important to at least partially compensate for these forces.

Broken tips will be permanently removed by means of a cleaving tool mounted in one of the substrate positions on the sample wheel. Therefore the AFM-chip features eight cantilevers for redundancy. The piezoresistors are contacted by wire-bonding and can be individually addressed via an external multiplexer. The AFM-chip is mounted on the scanner with two tilt angles relative to the substrate plane to ensure that only one tip at a time is in the lowest, imaging position. The cantilever tips are alternately equipped with monolithic silicon tips and CVD molded diamond tips.

The length of the cantilevers is alternately $580 \mu \mathrm{m}$ and or $610 \mu \mathrm{m}$, their width is $160 \mu \mathrm{m}$ and thickness about $8 \mu \mathrm{m}$. The spacing between two neighboring levers is $350 \mu \mathrm{m}$. Thus the whole chip has a width of at least $2.6 \mathrm{~mm}$. This implies directly, that simply breaking of the cantilevers for removing blunt tips is not sufficient: the edge of the chip at the first cantilever would touch the sample when measuring with the last few cantilevers. Therefore, the levers reside on rigid support beams rather than on the bulk of the chip. It is this support beam that is cleaved for cantilever removal (see fig. 3 ).

The space between two support beams, which have the full wafer thickness, is about $90 \mu \mathrm{m}$. Therefore, the standard $\mathrm{KOH}$ etching technique cannot be used for bulk-machining the AFMchip and must be replaced by anisotropic, deep reactive ion etching (DRIE) [4].

The electronic circuit for dynamic mode imaging is based on a PLL frequency-shift detection. The frequency shift of the oscillation is detected by keeping a constant phase difference between detection and driving signal. Thus, the phase transition value has to be specified during autonomous initialization of the AFM for dynamic mode operation. During this process cross-talk between cantilevers is of concern since the whole chip and therefore all cantilevers are excited at the same time. We expected a relatively strong coupling between neighboring levers. Therefore, two different lever lengths are used to separate their respective resonances by $6 \mathrm{kHz}$. This allows electronic detection of the desired peak within a reasonable frequency span without interference due to cross-talk by the neighboring cantilever. The choice of the correct phase transition can then be determined 


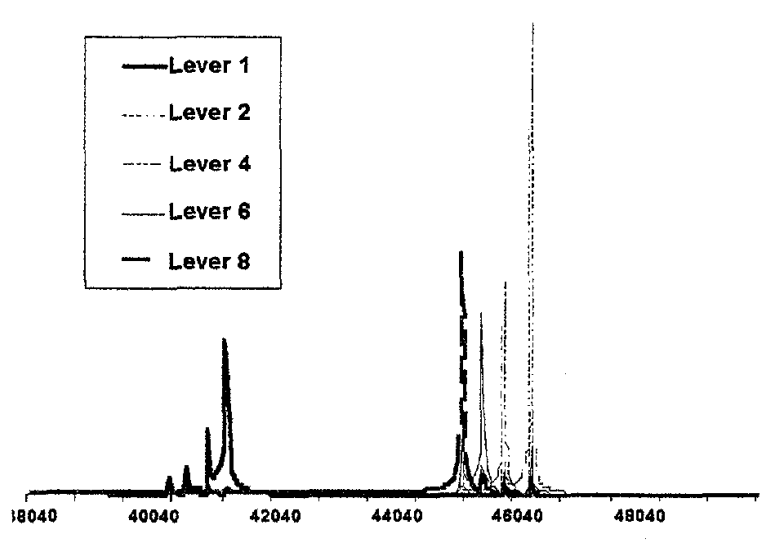

Figure 4. Simulated frequency response of the piezoresistors for cantilever 1 (low frequency) and cantilevers $2,4,6,8$ (high frequency). Cross-talk peaks induced by each cantilever on each cantilever.

autonomously by the software by simply cycling through all possible phase values and detecting the highest signal amplitude.

Finite element analysis of the array and experimental evaluation showed, however, that cross-talk is important not just between neighboring cantilevers but between all even numbered cantilevers $(2,4,6$ and 8$)$. We attribute this characteristic to the chip's asymmetry caused by the compensation sensor on the far right of the chip. Since these 4 cantilevers have approximately the same resonance frequency, the spectral response for each of them shows multiple peaks within a very narrow frequency span. It has been shown and tested that each of the peaks seen on the frequency response of a certain cantilever can be used as a reference for dynamic mode measurements.

However, changing pressures and temperatures on Mars might temporarily influence the cross-talk behavior and modify the amplitude distribution over the 4 peaks. Thus, the autonomous phase detection algorithm might detect a cross-talk induced phase shift that corresponds to a signal too low in amplitude, rendering dynamic measurements impossible. This problem could be overcome by having a more powerful autonomous algorithm for initializing the AFM in dynamic mode. For future designs, however, it is better to distribute the resonance frequencies of all 8 cantilevers evenly across the whole array. Figure 4 depicts the spectrum obtained by finite element analysis of a lever array. The analysis chip model has been created without a thermal compensation sensor and is symmetric. The cross-talk is present on all cantilevers.

The details of microfabrication are illustrated in the processflow chart in fig. 5. Silicon-on-insulator wafers are used as base material. The device layer has a thickness of $20 \mu \mathrm{m}$ and the support wafer is $380 \mu \mathrm{m}$ thick. In a first step (fig. 5a), $8 \mu \mathrm{m}$ high silicon tips are fabricated for every second cantilever by wet etching the device layer in $\mathrm{KOH}$ and using square thermal-oxide masks. The other four cantilevers are equipped with diamond tips later. Then phosphor-silicate glass (PSG) and silicon-oxide films are deposited in a chemical-vapor deposition (CVD) process. These films are then structured by photolithography and wet etching. The photoresist and the oxide were used as mask during (a)

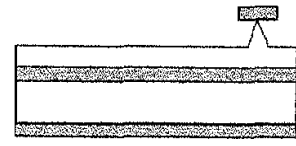

(b)

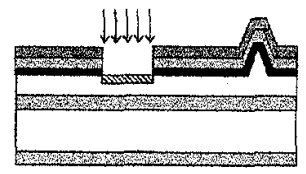

(c)

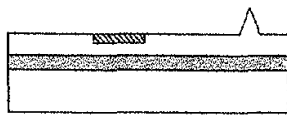

(d)

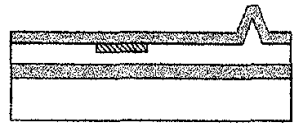

(e)

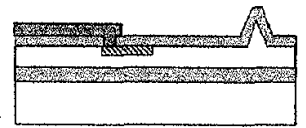

(f)

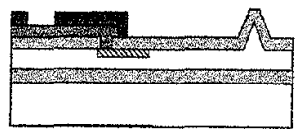

(g)

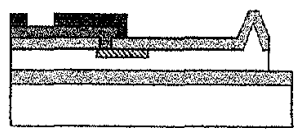

(h)

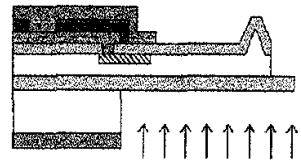

(i)

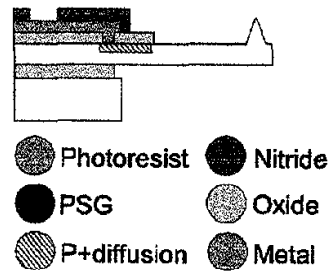

Figure 5. Process-flow chart of AFM chip:

a) Thermal oxide, lithography, oxide etching, KOH etching.

b) CVD PSG deposition, CVD oxide deposition, Lithography, Oxide etching, Implantation, removing resist, CVD oxide deposition, thermal treatment $\left(950^{\circ} \mathrm{C}, 30 \mathrm{~min}\right.$.).

c) Oxide etching.

d) CVD oxide deposition.

e) Lithography, Contact hole opening, Al evaporation, Lithography, Al etching.

f) Plasma SiN deposition, Lithography, SiN Plasma etching.

g) Lithography, Oxide etching, Topside Si etching.

h) Front side lithography, backside lithography, backside DRIE etching.

i) Oxided etching.

ion-implantation (fig. 5b). After stripping the mask, CVD oxide was again deposited, followed by a thermal treatment at $950 \mathrm{C}$ for 30 minutes fto activate the dopants. The oxide is replaced by a fresh layer for passivation and protection during subsequent processing (figs. $5 \mathrm{c}$ and $\mathrm{d}$ respectively). A third lithography structures a resist layer used as mask for wet etching the contact holes to the piezoresistors. A layer of aluminum is deposited and wet etched after the next lithography to define the contact paths and pads (fig. 5e). Figure $\mathbf{5 f}$ depicts the passivation process of the chip through deposition of a plasma nitride layer and a fifth lithography to structure it. This nitride structuring is used to open the contact pads. The oxide layer remaining on the silicon is structured by lithography and wet etching and used as a mask to define the top shape of the cantilevers (fig. $5 \mathrm{~g}$ ). Fig. 5h shows the last top lithography for removing the remaining oxide on the cantilevers. The top resist is also used as a protection layer for the backside deep reactive ion etching (DRIE) which defines the support beams and the final chip shape. For this backside etching, lithography on the back of the wafer is performed. DRIE allows etching through the full wafer with almost vertical side walls, which is important for maintaining a small pitch of $350 \mu \mathrm{m}$ in the lever array. The oxide layer underneath the cantilever is used as an etch stop. As a final step, the oxide remaining underneath the 

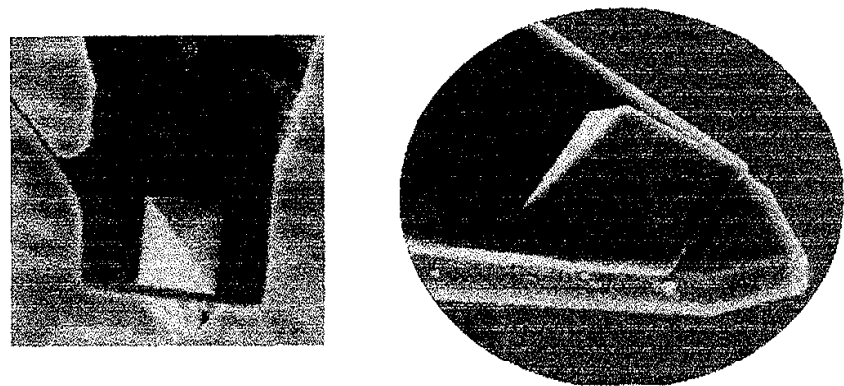

Figure 6. FIB Trimming of diamond tip to fit cantilever shape. The left image shows the diamond tip with its support frame glued on the cantilever before FIB trimming. The left image shows the diamond tip after having been trimmed by focussed ion beam.

cantilever is removed by wet etching (fig. $5 i$ ). After this step, the individual AFM chips are still connected to a silicon frame via thin bridges. They are easily broken for removing the chips.

The diamond tips are fabricated by CVD into pyramidal silicon molds [5]. The tips are then bonded to a special pyrex substrate. The Si mould can then be removed in $\mathrm{KOH}$. Mounting of the diamond tips on the cantilevers is performed next, working on a chip-by-chip base and using a micro-manipulator. Finally, parts of the diamond tip which jut the border of the cantilever are trimmed by a focused ion beam (Fig. $G$ ). The chip is directly glued to the scanner. The electrical contacts are wire bonded.

The mounted chip has withstood shocks up to $3000 \mathrm{~g}$ acceleration at $3 \mathrm{kHz}$, and the anticipated vibrations and thermal cycling of the mission.

\section{RESULTS}

Figures 7 and 8 show the image of a calibration grid $(10 \mu \mathrm{m}$ pitch) and a Mars-analog sample ( $\mathrm{Fe}_{2} \mathrm{O}_{3}$-particles), both measured with the Mars AFM system.

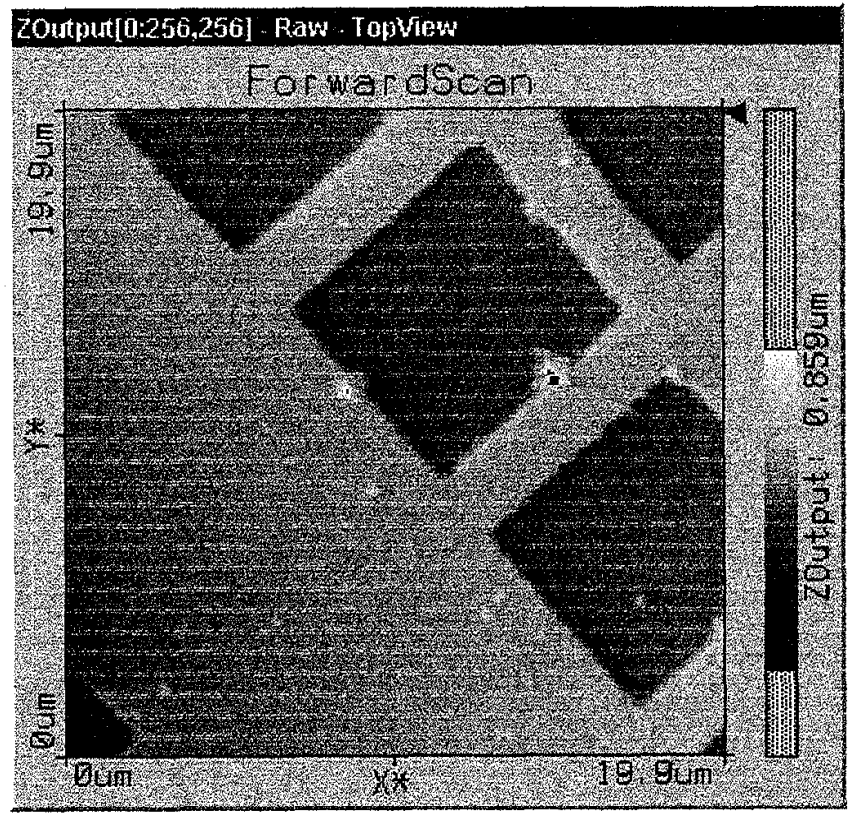

Figure 7. AFM dynamic mode image of a 10 um pitch calibration sample. The step of the grid is $200 \mathrm{~nm}$.

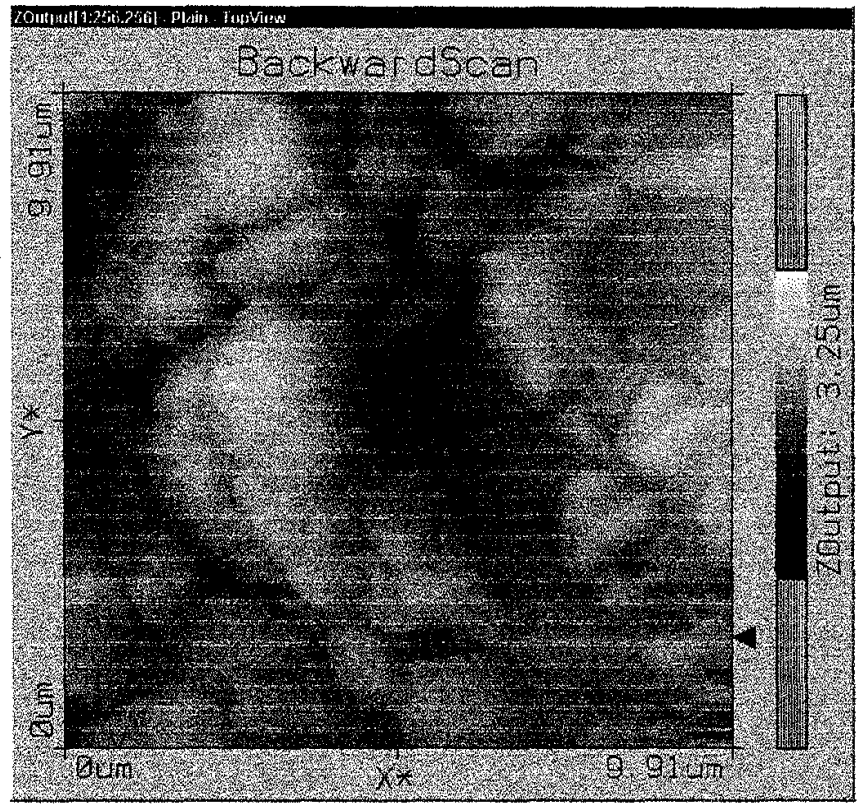

Figure 8. AFM dynamic mode image of Mars equivalent dust particles $\left(\mathrm{Fe}_{2} \mathrm{O}_{3}\right)$.

\section{ACKNOWLEDGEMENTS}

The work described was performed through support from the Swiss government's MINAST program and an award by NASA's Code U.

\section{REFERENCES}

1 W. J. Markiewicz, R. M. Sablotny, H. U. Keller, N. Thomas, D. Titiv, P. H. Smith, "Properties of Martian aerosols derived from Imager for Mars Pathfinder midday sky brightness data", J. Geophys Res - Planets, 104, 9009 (1999).

\section{M. Malin http://www.msss.com/mars-images/moc (2000).}

3 M. Tortonese, R.C. Barett, C.F. Quate, "Atomic resolution with an atomic force microscope using piezoresistive detection" Appl. Phys. Lett. 62, 834, (1993).

4. P.-A. Clerc, L. Dellmann, F. Grétillat, M.-A. Grétillat, P.-F. Indermuihle, $\mathrm{S}$. Jeanneret, $\mathrm{Ph}$. Luginbühl, C. Marxer, T.L. Pfeffer, G.-A. Racie, S. Roth, U. Staufer, C. Stebler, P. Thiébaud, and N.F de Rooij, "Advanced deep reactive ion etching: a versatile tool for microelectromechanical systems", J.Micromech. Microeng. 8, 272 (1998).

5. Niedermann, W. Hänni, S. Thurre, M. Gjoni, A. Perret, N. PH Skinner, P.-F. Indermithle, U. Staufer, N. F. de Rooij, "Mounting of micromachined diamond tips and cantilevers", Surf. and interface anal. 27, 296 (1999). 